\title{
RICERCA
}

\section{IL BAMBINO IN COMUNITÀ E LA DIFFUSIONE DI SARS-CoV-2: L'ESPERIENZA DEI CENTRI ESTIVI}

\author{
Simona Di Mario ${ }^{1}$, Elisabetta Mazzanti ${ }^{1}$, Giulio Matteo $^{2}$, Gino Passarini ${ }^{3}$, Maria Teresa Paladino ${ }^{3}$, \\ Giovanna Mattei ${ }^{2}$, Luca Barbieri ${ }^{1}$, per il Gruppo Collaborativo Covid-19 in Pediatria* \\ Direzione generale Cura della Persona, Salute e Welfare, ' Servizio Assistenza Territoriale, \\ ${ }^{2}$ Servizio Prevenzione Collettiva e Sanità Pubblica, ${ }^{3}$ Servizio Politiche Sociali e Socio-Educative \\ Regione Emilia-Romagna, Bologna \\ *Gruppo Collaborativo Covid-19 in Pediatria: Elisa Alberti, Antonella Antonelli, Anna Maria Baldoni, \\ Alessandro Ballestrazzi, Marcello Bergamini, Antonella Brunelli, Silvia Cattani, Tiziano Dall’Osso, \\ Alfredo Ferrari, Giuseppe Gregori, Annamaria Magistà, Romano Manzotti, Maddalena Marchesi, Sandra Mari, \\ Franco Mazzini, Gino Montagna, Valerio Moschettini, Luciana Nicoli, Carmelo Palmeri, Costantino Panza, \\ Rita Ricci, Roberto Sacchetti, Stefano Testi, Aldo Vinattieri, Alessandro Volta
}

Indirizzo per corrispondenza: simona.dimario@regione.emilia-romagna.it

\section{CHILDREN AND SARS-CoV-2 SPREAD: THE EXPERIENCE IN SUMMER CAMPS}

\section{Key words}

Covid-19, Children, Summer camp, Monitoring

\begin{abstract}
Data on SARS-CoV-2 in children are sparse and sometimes contradictory. In Italy the closing of the school at the end of February 2020 up to June probably contributed to slow the epidemic, but was also associated with an increased risk of mental and physical disruption in children and adolescents. In the Emilia-Romagna Region summer camps were opened starting from the first week of June, even in absence of clear and univocal guidelines. Opening summer camps gave the opportunity to test the health and the educational systems in general and, in particular, the effectiveness of the implemented preventive measures and the test, treat and track system, therefore to increase the knowledge on Covid-19 in Paediatrics. The paper describes the building of a virtual space for collaboration with family paediatricians, community paediatricians, public health departments, preschool departments and the monitoring of the health conditions in children attending summer camps. This model can help guide health care decision with the reopening of schools.
\end{abstract}

\section{RIASSUNTO}

I dati relativi al rischio nei bambini di infettarsi con il nuovo coronavirus, di sviluppare una malattia più o meno grave e di diffondere l'infezione sono rari e a volte con- traddittori. La scelta di chiudere le scuole a fine febbraio 2020 e di non riaprirle fino al termine dell'anno scolastico in giugno ha probabilmente contribuito a ridurre la circolazione del virus, ma allo stesso tempo ha esposto i bambini/ragazzi a un aumentato rischio di malessere e disagio psico-fisico. In Emilia-Romagna l'apertura dei centri estivi fin dalla prima settimana di giugno, anche in assenza di chiare e univoche linee guida, è stata l'occasione per sperimentare la tenuta del Sistema sanitario ed educativo, la capacità di applicare le misure di prevenzione e controllo, e conoscere meglio l'epidemiologia di SARS-CoV-2 in età pediatrica. In questo articolo descriviamo la nostra esperienza nel costruire uno spazio virtuale di collaborazione con la Pediatria di famiglia, la Pediatria di comunità, la Sanità pubblica, in un lavoro di coordinamento che ha coinvolto i Servizi educativi e sanitari sul territorio e il Sistema regionale e che ha previsto la raccolta di dati sulle condizioni di salute dei bambini che hanno frequentato i centri estivi. Questo modello può aiutare a indirizzare le scelte sanitarie con la riaperture degli asili e delle scuole.

\section{INTRODUZIONE}

La comparsa in Italia del virus SARS-CoV-2, agente causale di Covid-19, con i primi casi autoctoni identificati a fine febbraio in Lombardia e dopo pochi giorni in Emilia-Romagna, ha causato la progressiva implementazione di misure preventive per contrastare la diffusione dell'epidemia, con interventi che hanno riguardato anche la chiusura delle scuole e degli spazi educativi (a partire dal 24 febbraio in Emilia-Romagna) ${ }^{1}$. Il conseguente periodo di chiusura ha verosimilmente contribuito alla riduzione dei casi e della trasmissione virale ${ }^{2}$, permettendo 
così l'avvio della cosiddetta Fase 2, con progressiva riapertura delle attività commerciali e degli spostamenti fra Regioni da metà maggio, mentre le attività educative e scolastiche hanno continuato a rimanere chiuse ${ }^{1}$. I primi studi pubblicati sui casi di Covid-19 in età pediatrica suggerivano che, in questa fascia di età, l'infezione da SARSCoV-2 è probabilmente meno frequente ${ }^{3,4}$, che la sintomatologia è comunque più lieve e che, in assenza di comorbidità, sono rarissimi i casi con quadro clinico grave e morte ${ }^{5,6}$. Sporadiche segnalazioni hanno fatto ipotizzare anche un ruolo di minore trasmissione dell'infezione dal bambino all'adulto ${ }^{7,8}$. Il quadro però è ancora poco definito e probabilmente influenzato dalla minore possibilità di socializzazione dei bambini nella fase di chiusura. Il ruolo dei bambini come diffusori di malattia Covid-19 è stato messo in discussione ${ }^{9-12}$, anche in una visione più ampia a tutela del benessere di questa fascia di popolazione, minoranza numerica i cui interessi sono raramente rappresentati nel dibattito politico e culturale.

Con l'obiettivo di definire il rischio di circolazione del virus e di contagio dei bambini quando inseriti nuovamente in comunità, e di coordinare le attività di assistenza clinica e sorveglianza necessarie, la Regione EmiliaRomagna ha organizzato, in collaborazione con i pediatri di famiglia (PdF) del proprio territorio, un monitoraggio per verificare il numero di casi sintomatici, di tamponi richiesti e di tamponi positivi fra $\mathrm{i}$ bambini che a partire da metà giugno e fino alla fine di agosto hanno frequentato i centri estivi, quei luoghi, cioè, di aggregazione, educazione e socialità gestiti dagli Enti locali o dal Terzo settore, che durante l'estate offrono alle famiglie un servizio alternativo a quello dei Servizi educativi e scolastici.

\section{METODO}

Prima della riapertura dei centri estivi nel territorio della Regione Emilia-Romagna (il 6 giugno 2020) si sono tenuti 4 incontri in videoconferenza con una rappresentanza di pediatri (di famiglia e di comunità) e dei Servizi regionali sanitari e sociali per definire le procedure più adatte a garantire la ripresa in sicurezza delle attività. Ai $\mathrm{PdF}$ è stato proposto di partecipare a un'indagine prospettica: è stato messo a disposizione un questionario on-line per la raccolta settimanale di un numero contenuto di variabili riportate in maniera aggregata e completamente anonima (Tabella I). I questionari compilati venivano automaticamente inviati al Coordinamento regionale. I dati sono stati analizzati ed è stato inviato un report con le informazioni raccolte ai pediatri partecipanti. I dati raccolti sono stati confrontati con quelli del Sistema di Sorveglianza in funzione presso i Dipartimenti di Sanità Pubblica (DSP) aziendali della Regione, che hanno inviato settimanalmente dati aggregati relativi a numero di tamponi effettuati sul personale dei centri estivi e sui bambini partecipanti ai centri estivi e relativo esito (la specifica dei centri estivi è stata attivata $a d h o c$ ). I risultati dei questionari sono riportati come numeri e frequenze.

\section{DOMANDE CONTENUTE NEL QUESTIONARIO}

1. Azienda USL di appartenenza

2. Settimana di riferimento

3. Numero di visite per sintomatologia acuta in bambini che frequentano i centri estivi

4. Numero di tamponi richiesti per bambini che frequentano i centri estivi

5. Numero di tamponi positivi in bambini che frequentano i centri estivi

6. Numero di famiglie che rifiutano di eseguire il tampone

\section{Tabella I}

\section{RISULTATI}

Fra il 6 giugno e il 30 agosto 2020 hanno inviato informazioni relative ai bambini che frequentano i centri estivi 199/597 PdF, pari a 33\% del totale (dato di partecipazione media settimanale), con una variazione compresa fra 118 e 324 pediatri a settimana. Sono state effettuate complessivamente 1436 visite a bambini che frequentano i centri estivi e che hanno sviluppato sintomi acuti (media settimanale 144 visite). I tamponi richiesti ed eseguiti sono stati 637 (media settimanale di 64 tamponi): nessuno dei tamponi richiesti dai PdF per sintomatologia acuta è risultato positivo. Due test, poi risultati positivi, sono stati eseguiti nell'ambito di attività di contact tracing dal DSP (figli di genitore positivo, contagiato presumibilmente sul luogo di lavoro). Sono state 7 , infine, le famiglie che si sono rifiutate di fare sottoporre il bambino al tampone.

Nello stesso periodo i dati raccolti dai DSP delle Aziende hanno registrato 18 tamponi positivi in personale e fruitori dei centri estivi, rispettivamente 9 fra gli operatori e 9 fra i bambini; in un solo caso il luogo di presunta esposizione è stato il centro estivo.

Una valutazione dell'Associazione Nazionale dei Comuni Italiani (ANCI) sezione Emilia-Romagna stima che siano stati oltre cinquantamila $i$ bambini che hanno partecipato a uno degli oltre duemila centri estivi attivati in Regione quest'anno.

\section{DISCUSSIONE}

Ad aprile 2020 le indicazioni relative alla frequenza di scuole e spazi educativi per $\mathrm{i}$ bambini in relazione all'epidemia di Covid-19 erano rare ed essenzialmente basate su opinioni ${ }^{13,14}$. La chiusura delle scuole è stata più breve o addirittura non è mai avvenuta nei Paesi del Nord Europa; in altri Paesi, come l'Italia, le scuole sono rimaste chiuse fino alla fine dell'anno scolastico. In questo quadro generale di incertezza, la ripresa delle attività dei centri estivi in Emilia-Romagna ha richiesto un lavoro di coor- 
dinamento fra livello centrale e periferico, sia in ambito sanitario che educativo, particolarmente intenso. Il confronto periodico tramite videoconferenza avviato ad aprile ha consentito di ripartire condividendo protocolli e procedure comuni, finalizzate a contenere al minimo il rischio di contagio - pur nella consapevolezza dell'impossibilità di azzerare il rischio - e allo stesso tempo di far tornare $i$ bambini a una dimensione di socialità necessaria al loro benessere $^{9-12}$.

Essersi dati uno strumento semplice di monitoraggio (tempo medio di compilazione inferiore a due minuti), online, ha consentito di delineare un quadro che, seppure parziale (dal $20 \%$ al $54 \%$ dei PdF ha partecipato alla raccolta e invio di dati nelle varie settimane), è però informativo: le visite per episodi di acuzie non sono state molte rispetto a quanto avveniva solitamente durante il periodo della frequenza dei centri estivi (data qualitativo raccolto durante gli incontri): è possibile che l'isolamento dovuto alla permanenza forzata in casa nei mesi del lockdown, oltre all'applicazione delle misure di prevenzione, abbia ridotto la circolazione di patogeni fra i bambini.

Nessuno dei 637 tamponi richiesti in bambini sintomatici in cui il PdF sospettava l'infezione da SARS-CoV-2 (quasi un bambino sintomatico ogni due visitati è stato sottoposto a tampone) è risultato positivo. Gli unici due casi di positività in bambini frequentanti i centri estivi sono stati in soggetti asintomatici tracciati dal DSP nell'ambito di attività di contact tracing. Questo dato suggerisce che, anche dopo due mesi di ripresa delle attività in comunità, la circolazione di SARS-CoV-2 in età pediatrica è ancora sostanzialmente associata all'esposizione intrafamiliare. È possibile che quanto osservato sia legato all'attento rispetto delle norme di prevenzione concordate con gli Enti gestori e attuate durante questo periodo nei centri estivi (stipula di un Patto di corresponsabilità fra Enti gestori e famiglie rispetto alle procedure da seguire e all'osservazioni di norme di prevenzione concordate, procedure di triage in ingresso, lavoro in piccoli gruppi stabili, periodica igienizzazione degli spazi utilizzati, norme per la ristorazione ecc.) ${ }^{15,16}$. È anche possibile che la mobilità delle persone, e quindi la circolazione del virus, sia stata molto contenuta nei mesi di giugno e luglio (ad agosto il numero di centri estivi attivi è, come di consuetudine, ridotto). Eppure, monitorare questi dati e restituire un feedback ai PdF è stato utile per iniziare a costruire un'esperienza basata maggiormente sui dati e meno sulle opinioni rispetto alla gestione dei bambini in epoca Covid-19: il livello di preoccupazione percepito durante le prime videoconferenze con i professionisti è sicuramente andato riducendosi nel tempo, alla luce del confronto fra pari e dei dati raccolti.

L'esperienza acquisita sarà la base per un sistema di monitoraggio da attivare con la ripresa delle scuole di cui sia i PdF che i decisori politici, ma anche le scuole e le famiglie, sentono fortemente la necessità: è prevedibile che con l'autunno ci sarà una differente e probabilmente maggiore circolazione del virus, dovuta alla più elevata mobilità delle persone $\mathrm{e}$ intensificazione delle attività produttive. Il ruolo della riapertura delle scuole sulla circolazione del virus non è noto, ma è verosimile che inciderà, anche se solo marginalmente. Modelli previsionali indicherebbero che le scuole influiscono solo per il $2 \%$ rispetto alla circolazione di SARS-CoV- $2^{17}$, anche se non tutti concordano con queste valutazioni ${ }^{18}$.

Nella convinzione che i vantaggi della riapertura delle scuole superino comunque gli eventuali problemi legati all'aumento di circolazione del virus ${ }^{19-21}$, pur consapevoli della complessità dei sistemi e dei differenti interessi in gioco $^{22}$, ci sembra che il confronto periodico con i professionisti coinvolti e la disponibilità di dati localmente raccolti - rappresentativi quindi della circolazione virale locale - con feedback periodici e ravvicinati (bisettimanali) possa consentire:

1. ai PdF di modulare la propria attività diagnostica e assistenziale,

2. ai Servizi di regolare la propria capacità di risposta, anche anticipando le richieste in base ai trend evidenziati,

3. ai decisori di rafforzare o al contrario di rilassare le misure preventive e di indirizzare i messaggi informativi rivolti alla popolazione.

Il monitoraggio sarà uno degli strumenti che ci aiuterà ad affrontare meglio questo periodo, con l'obiettivo di limitare al minimo le interruzioni delle attività scolastiche.

Conflitti di interesse: nessuno.

\section{Ringraziamenti}

Si ringraziano gli oltre trecento pediatri di famiglia della Regione Emilia-Romagna che hanno partecipato all'indagine.

\section{BIBLIOGRAFIA}

[1] Pandemia di Covid-19 del 2020 in Italia. Wikipedia: The Free Encyclopedia. Wikimedia Foundation, Inc., 22 July 2004. Ultimo accesso: 3 settembre 2020 .

[2] Coronavirus in Italia, i dati e la mappa. Il trend settimanale. Il Sole 24 Ore. Ultimo accesso: 3 settembre 2020 .

[3] Lavezzo E, Franchin E, Ciavarella C, et al.; Imperial College Covid-19 Response Team. Suppression of a SARS-CoV-2 outbreak in the Italian municipality of Vo'. Nature 2020;584(7821):425-9.

[4] CDC Covid-19 Response Team. Coronavirus disease 2019 in children - United States, February 12-April 2, 2020. MMWR Morb Mortal Wkly Rep 2020;69:422-6.

[5] World Health Organization. Report of the WHO China joint mission on coronavirus disease 2019 (Covid-19). 16-24 febbraio 2020. Ultimo accesso: 3 settembre 2020 . 
[6] Dong Y, Mo X, Hu Y, et al. Epidemiology of Covid-19 among children in China. Pediatrics 2020; 145:e20200702.

[7] Danis K, Epaulard O, Bénet T, et al; Investigation Team. Cluster of coronavirus disease 2019 (Covid19) in the French Alps, February 2020. Clin Infect Dis 2020;71:825-32.

[8] RIVM. National Institute for Public Health and the Environment. Ministry of Health, Welfare and Sport. Children and schools. Ultimo accesso: 3 settembre 2020.

[9] Marchetti F, Guiducci C. Covid-19 e bambini: le due facce di una diversa medaglia. Medico e Bambino 2020;4:219-21.

[10] Tamburlini G, Marchetti F. Pandemia di Covid-19: motivazioni e indicazioni per l'apertura di spazi educativi per bambini. Medico e Bambino 2020;39 (5):301-4.

[11] Esposito S, Principi N. School closure during the coronavirus disease 2019 (Covid-19) pandemic: an effective intervention at the global level? JAMA Pediatr 2020 May 13 [Epub ahead of print].

[12] Munro APS, Faust SN. Children are not COVID19 super spreaders: time to go back to school. Arch Dis Child 2020;105(7):618-9.

[13] Centre for Disease Prevention and Control. Guidance for childcare. Ultimo accesso: 3 settembre 2020.

[14] Ministero della Salute danese. Inspiration og hjælp til medarbejdere i dagtilbud for børn 0-6 år [Ispirazione e aiuto per i dipendenti nell'asilo nido per bambini 06 anni]. Ultimo accesso: 3 settembre 2020.
[15] Regione Emilia-Romagna, Decreto del presidente num. 95 del 01/06/2020. Protocollo regionale per attività ludico-ricreative - centri estivi - per i bambini e gli adolescenti dai 3 ai 17 anni. Bologna.

[16] Regione Emilia-Romagna, Decreto del presidente num. 111 del 15/06/2020. Protocollo regionale per avvio di attività estive specificamente dedicate a bambine e bambini dai 9 ai 36 mesi di età. Bologna.

[17] Viner RM, Russell SJ, Croker H, et al. School closure and management practices during coronavirus outbreaks including Covid-19: a rapid systematic review. Lancet Child Adolesc Health 2020;4:397-404.

[18] Lin PI, Chen YC. Debates around the role of school closures in the coronavirus 2019 pandemic. JAMA Pediatr 2020 Aug 31.

[19] Joseph SJ, Bhandari SS, Ranjitkar S, Dutta S. School closures and mental health concerns for children and adolescents during the Covid-19 pandemic. Psychiatr Danub 2020;32(2):309-10.

[20] Baron EJ, Goldstein EG, Wallace CT. Suffering in silence: how Covid-19 school closures inhibit the reporting of child maltreatment. J Public Econ 2020; 190:104258.

[21] Marchetti F. Gli effetti avversi del Covid-19 in età pediatrica: la pandemia secondaria. Medico e Bambino pagine elettroniche 2020;23(6):115-7.

[22] Pramling Samuelsson I, Wagner JT, Eriksen Ødegaard E. The coronavirus pandemic and lessons learned in preschools in Norway, Sweden and the United States: OMEP Policy Forum. Int J Early Child 2020;17:1-16. 